\title{
Characterization of lignin
}

Mikayla McKenna-Pettit ${ }^{1}$, Neda Nazemifard ${ }^{1}$, Niloufar Manafi ${ }^{1}$

${ }^{1}$ Department of Chemical and Materials Engineering, University of Alberta

\section{Abstract}

Lignin is a complex organic compound crucial to the structural tissues of vascular plants, such as trees. The cyclic structure and aromaticity of lignin give it significant potential to be used as a renewable and safe replacement for toxic aromatic compounds in chemical and industrial processes. The purpose of this experiment was to characterize lignin, specifically the particle diameter and zeta potential, using both the Zetasizer Nano ZSP and the ImageJ image processing software, and to compare the accuracy of both measurement methods. Due to the natural fluorescence of lignin, a fluorescence microscope is used to capture images of lignin particles. By having a known distance and the scale of measurement, with ImageJ it is possible to calculate distances, such as the diameters of particles in images. The Zetasizer Nano ZSP is a device capable of measuring both particle diameter and zeta potential, which is the electrical charge existing on particles suspended in a medium. Small amounts of lignin, in powder form, are placed into scintillation vials with different amounts of distilled water to create 3 different concentrations of sample to measure using the Zetasizer. Between $10-15 \mathrm{~mL}$ of sample are placed into specialized measurement cells and put into the Zetasizer. Multiple measurements are conducted and averaged to achieve accurate results. The measured zeta potential value is indicative of the stability of the lignin. If the value of zeta potential is higher than $30 \mathrm{mV}$, negative or positive, it has a high stability and low reactivity. The average values of zeta potential measured by the Zetasizer ranged from $-27 \mathrm{mV}$ to $-21 \mathrm{mV}$. The particle diameter is important in characterization, as the smaller and more distributed the particles are, there is a larger surface area for reactions to occur. Average particle diameter measured by the Zetasizer ranged from $926-976 \mu \mathrm{m}$. The results from the Zetasizer Nano ZSP are more accurate than those from the ImageJ software, as ImageJ allows for a substantial amount of human error to impact the results. The outcomes help direct future experiments using lignin and beneficial to future research concerning lignin and its potential.

Key words:

lignin, characterization, zeta potential, particle diameter

Cite as: McKenna-Pettit, M., Nazemifard, N., Manafi, N. 2019. Characterization of lignin. Alberta Academic Review, Vol 2 (2) 55-56, WISEST Special Issue (non peer-reviewed), DOI 10.29173/aar63. 
McKenna-Pettit et al., 2019

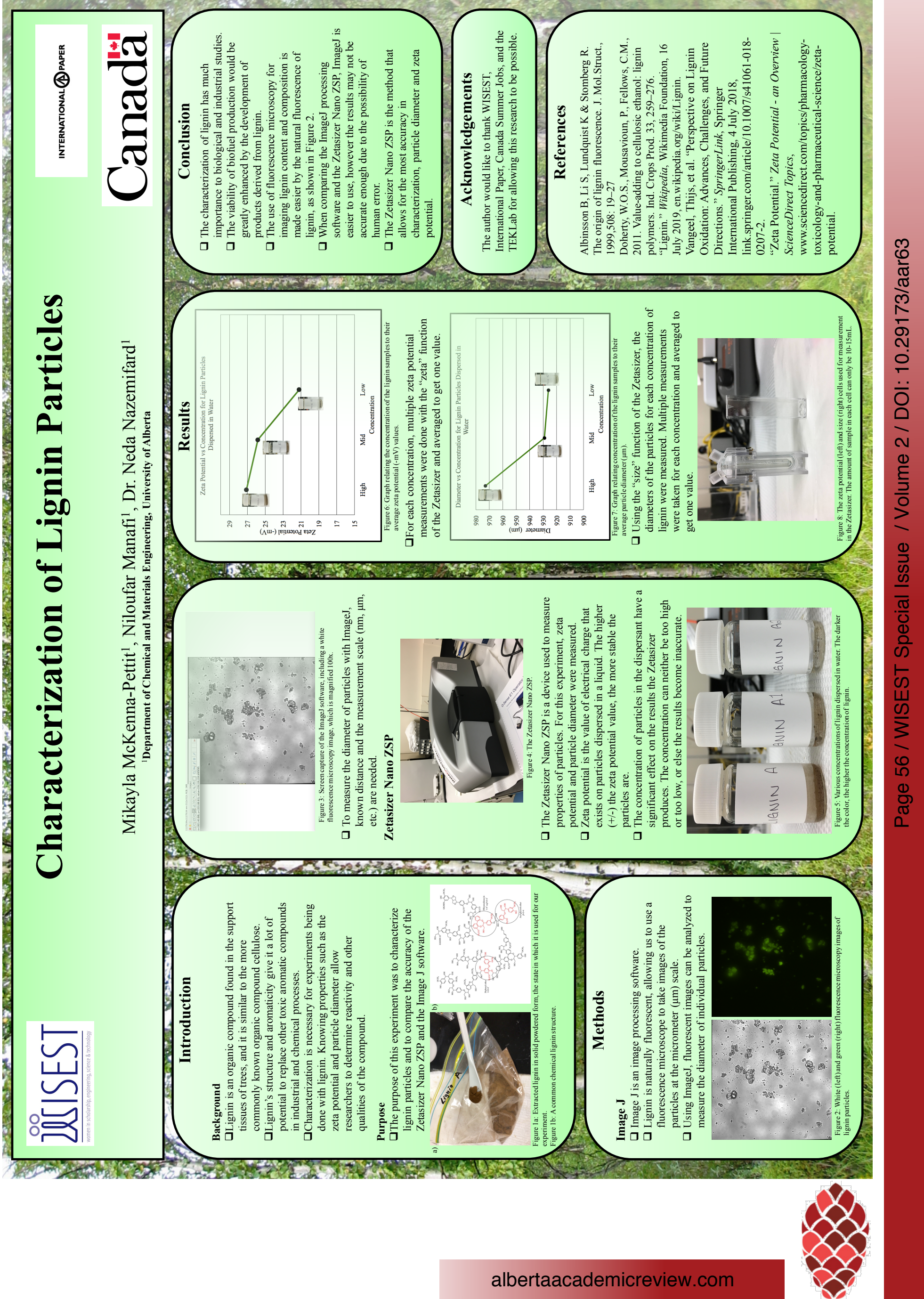

